\title{
Study the effect of gamma ray on silver nanoparticles prepared by pulse laser ablation in liquid technique (PLAL)
}

\author{
Mohammed Maher Al-Maher ${ }^{1 *}$, Najwa I. Al-Barhawi², Mushtaq Abed Al-Jubbori ${ }^{3}$ \\ ${ }^{1 *}$ Department of Physics, College of Education for Pure Sciences, University of Mosul, Mosul, Iraq \\ ${ }^{2}$ Directorate of Education in Nineveh, Ministry of Education, Mosul, Iraq. \\ ${ }^{3}$ Department of Biology, College of Education for Pure Science, Mosul University, Mosul, Iraq. \\ E-mail: ${ }^{1 *}$ m_almaher90@yahoo.com, ${ }^{2}$ dr.najwa@uomosul.edu.iq, ${ }^{3}$ mushtaq_phy@uomosul.edu.iq
}

(Received January 08, 2021; Accepted February 10, 2021; Available online June 01, 2021)

DOI: 10.33899/edusj.2021.129320.1136, (c) 2021, College of Education for Pure Science, University of Mosul.

This is an open access article under the CC BY 4.0 license (http://creativecommons.org/licenses/by/4.0/).

\begin{abstract}
Silver nanoparticles in this study were prepared using the method of laser ablation in liquids (PLAL) at two energies (540 and 700) $\mathrm{mJ}$ and the count of pulses were (100,200,300 and 400) pulse. The prepared samples were divided into three groups: the first group of the samples were kept as they are without any irradiation but the second and third group were irradiated by Co-60 source with two amount of doses (7.5 and 11) KGy respectively. A series of measurements and studies were done , and from the pictures of Transmitted Electron Microscope (TEM), it was observed that the prepared nanoparticles at laser energy (540) $\mathrm{mJ}$ with pulses count (400) pulse were spherical but the prepared nanoparticles at laser (700) $\mathrm{mJ}$ with pulses cont (400) pulse were nano rods shape. The gamma irradiation effect was studied through its effect on the optical properties of the nanoparticles at different preparation conditions, represented by a change in the pulses and the energy of the laser used. The results showed that there was an increase the absorbance peaks when increasing the irradiated dose. The (TEM) image showed we get spherical nanoparticles in shape and nano composition. The average diameter of the prepared silver nanoparticles at (540) $\mathrm{mJ}$ laser energy in the range of $(30-40) \mathrm{nm}$.
\end{abstract}

Keywords: Optical properties, Nano particles, Silver nano, Gamma ray, Laser, TEM

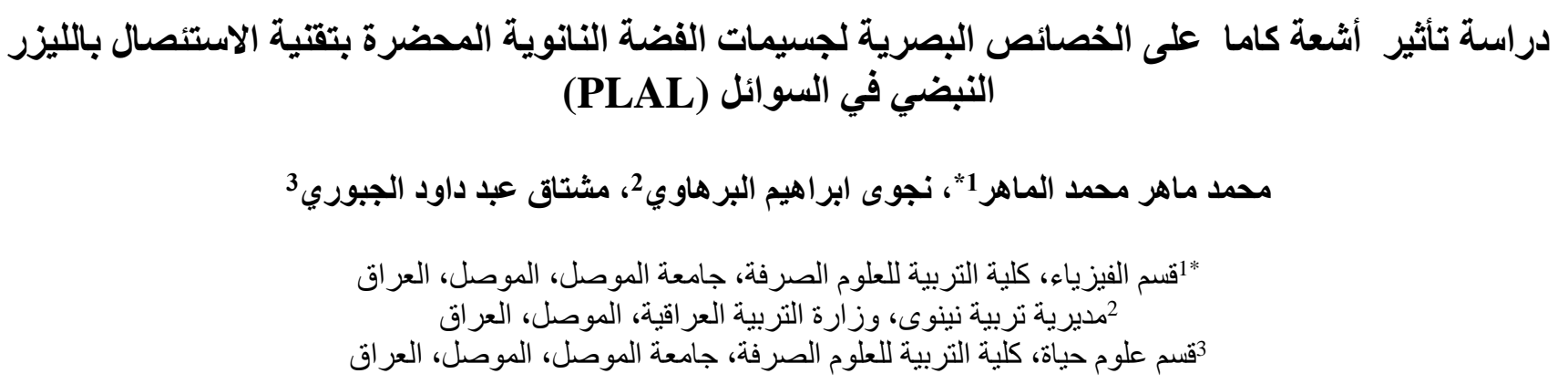

تم تحضير جسيمات الفضة النانوية باستعمال طريقة الاستئُسال بالليزر النبضي في السو ائل، بطاقات mJ (540, 700) وبعدد من

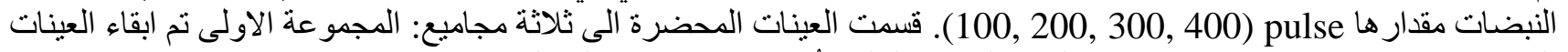

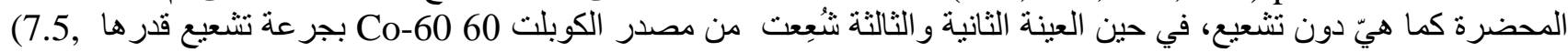
kGy (11. اجريت سلسلة من القياسات والدراسات ومن خلال صور المجهر الإلكتروني النافذ (TEM) لوحظ انه هنالك جسيمات 


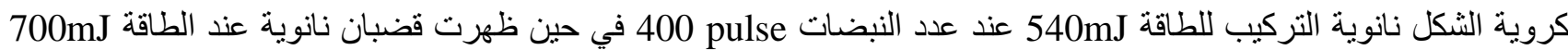

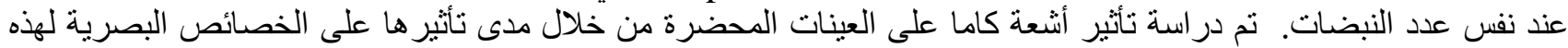

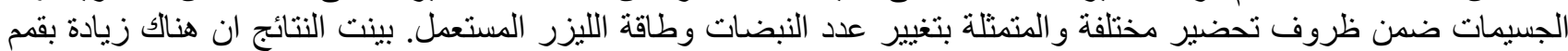

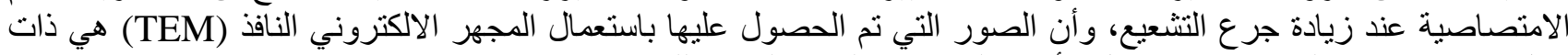

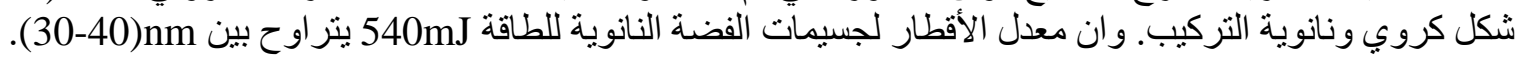

الكلمات المفتاحية: الخصائص البصرية، جسيمات النانو، الفضة النانوية، اشعة كاما، الليزر، المجر الالكتروني النافذ

تقدم تكنولوجيا النانو اليوم فرصاً محتملة لخلق مو اد ومنتجات أفضل. كما أن تكنولوجيا النانو لايها القدرة على تحسين البيئة، سواء

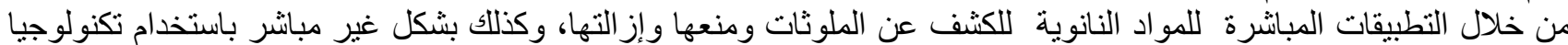

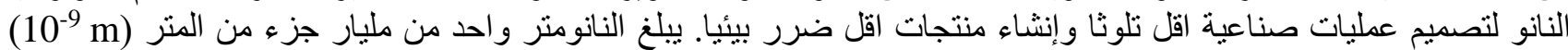

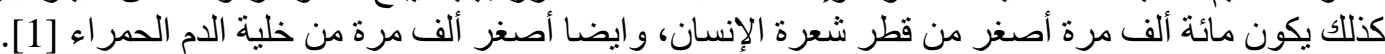

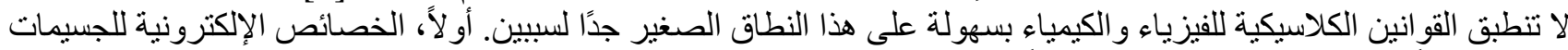

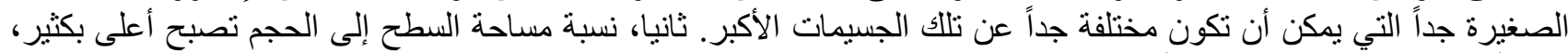

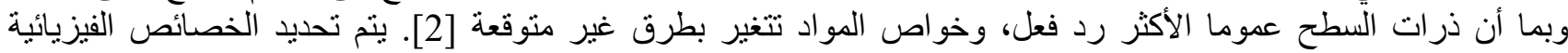

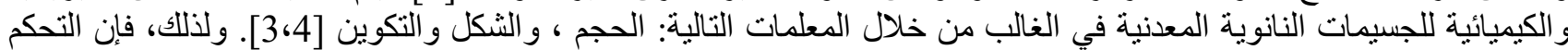

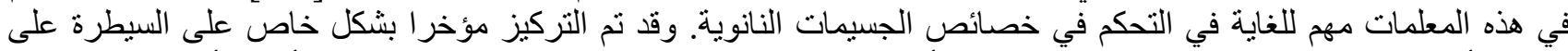

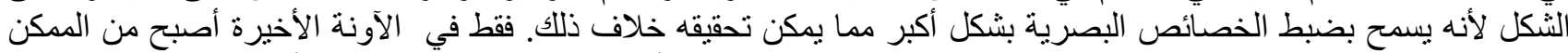

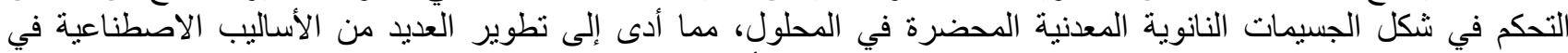

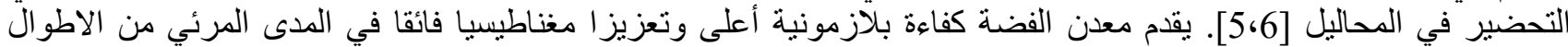

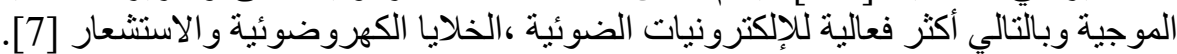

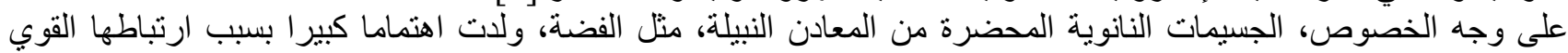

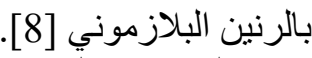

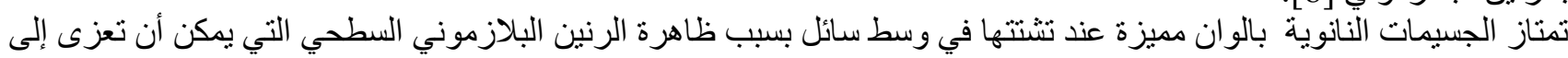

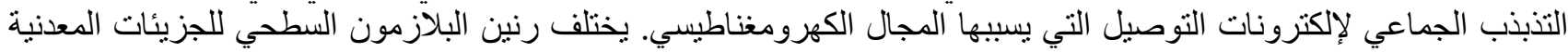

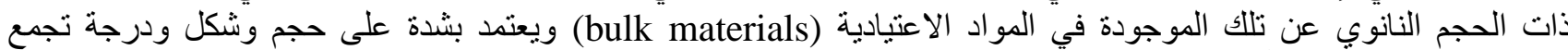

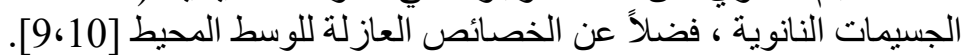

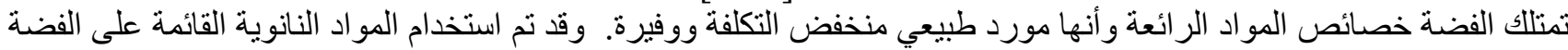

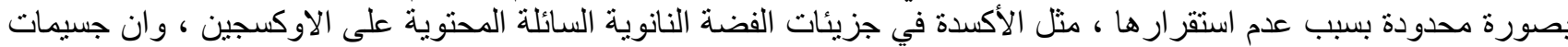

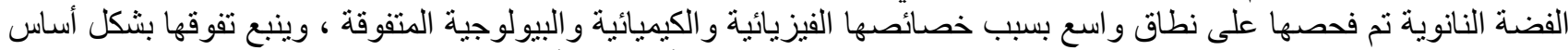

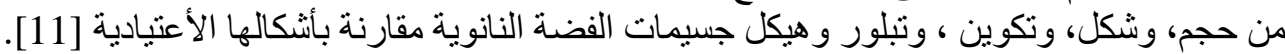

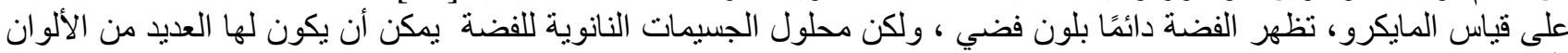

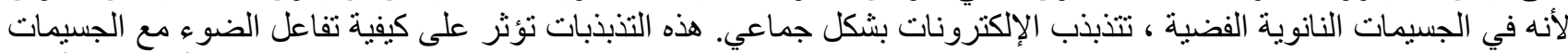

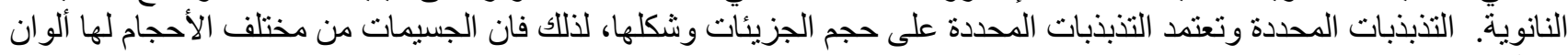

مختلفة [12].

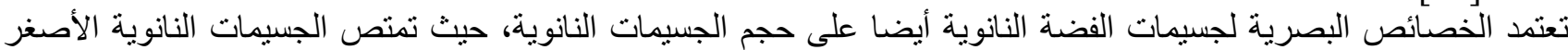

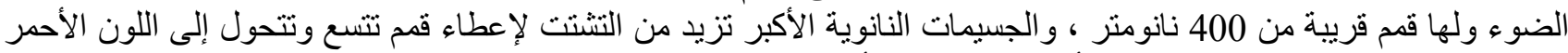

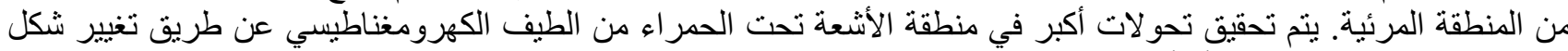

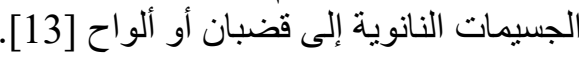

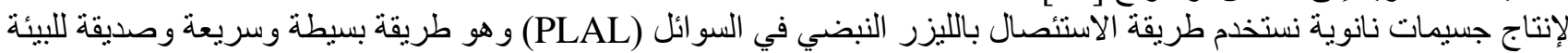

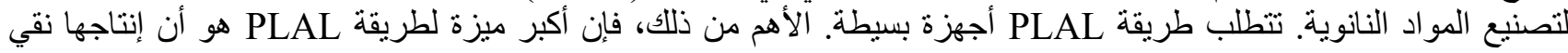

كيميائيا [14].

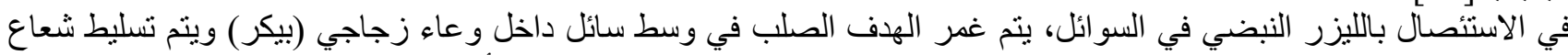

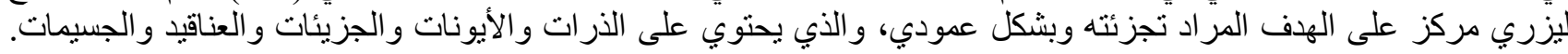

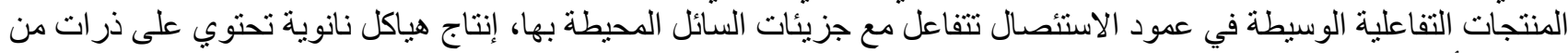

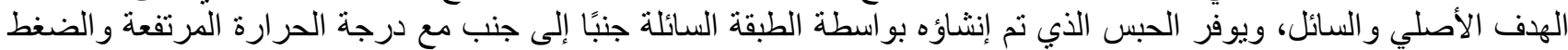

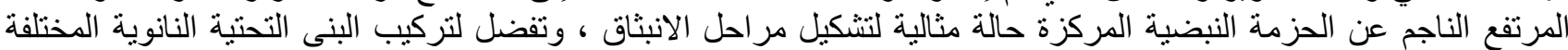




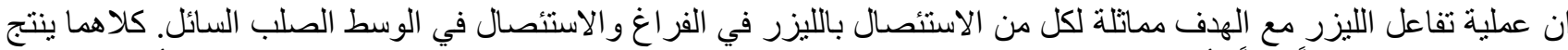

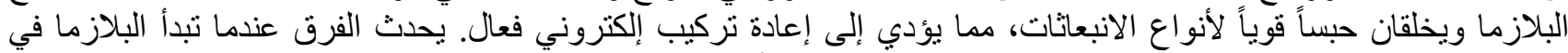

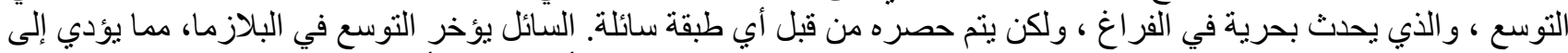

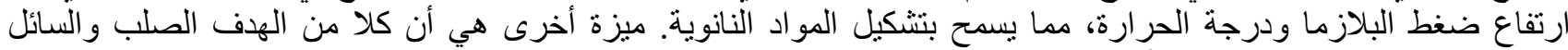

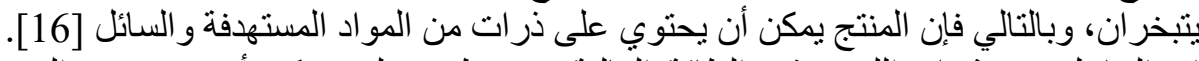

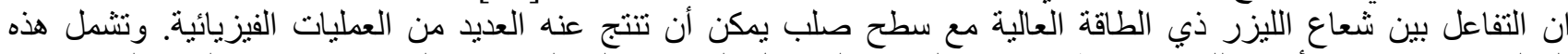

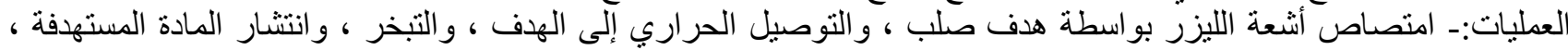

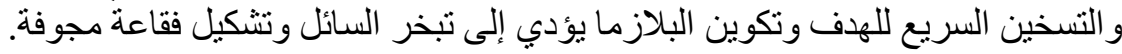

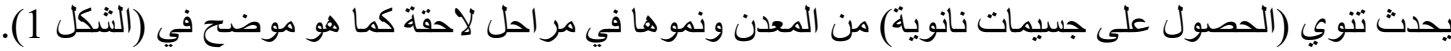
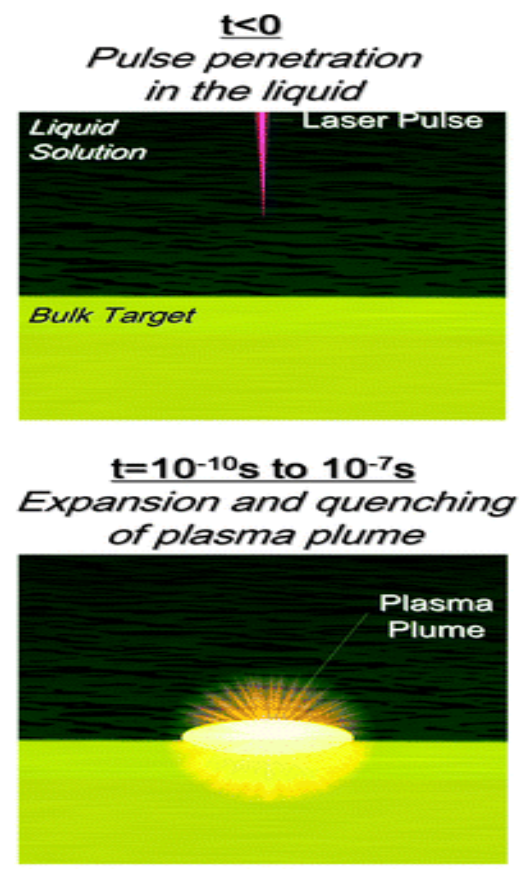

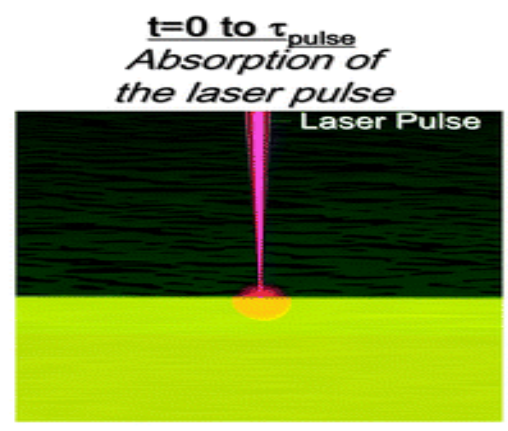

$t=10^{-6} s$ to $10^{-4} s$ Expansion and collapse of cavitation bubble

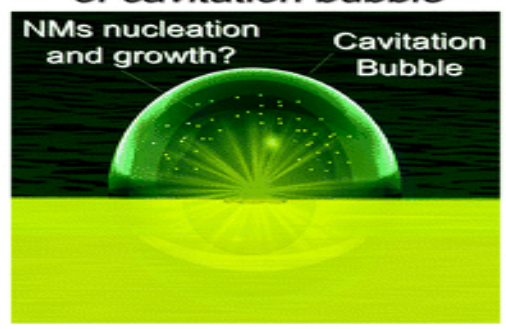

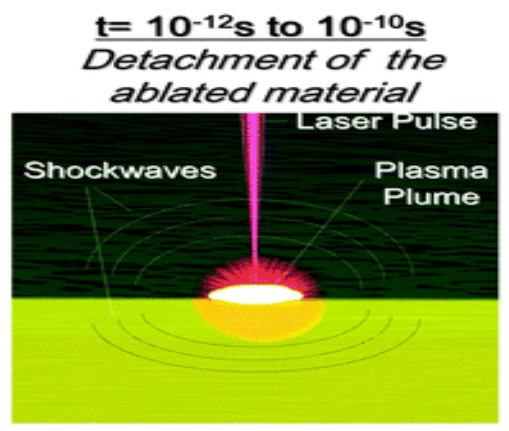

$\underline{t>10^{-4} s}$

Slow growth and agglomeration of NMs NMs

agglomeration

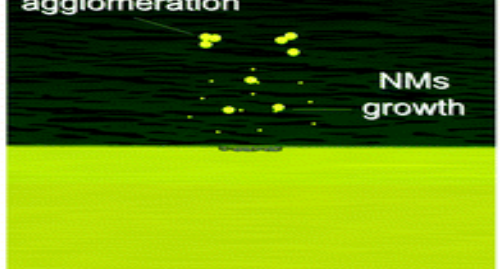

الثكل (1): مخطط يبين خطوات تحضير الجسيمات النانوية من خلال عملية الاستئصال بالليزر النبضي [17].

ان عملية الحصول على اشعة كاما تككن في الغالب من مصادر صناعية أثناء عملية التحلل النووي نتيجة لعودة النوى من حالة

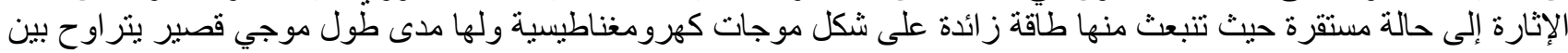

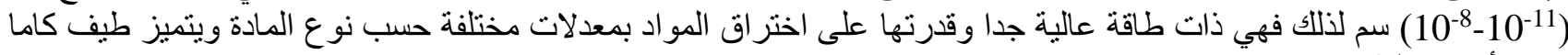
بكونه أحادي الطاقة [18]

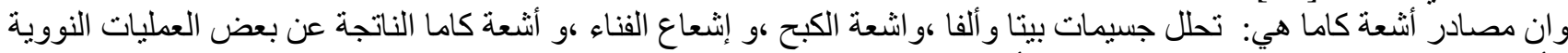

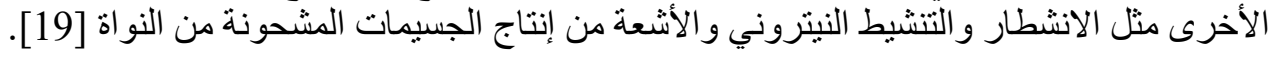

$$
\text { الجاتب العملي }
$$

يبين هذا الجانب كيفية الحصول على جسيمات الفضة النانوية باستعمال تقنية الاستئصال بالليزر النبضي في السو ائل، وكما موضحة في المخطط المبين بالثكل (2). 


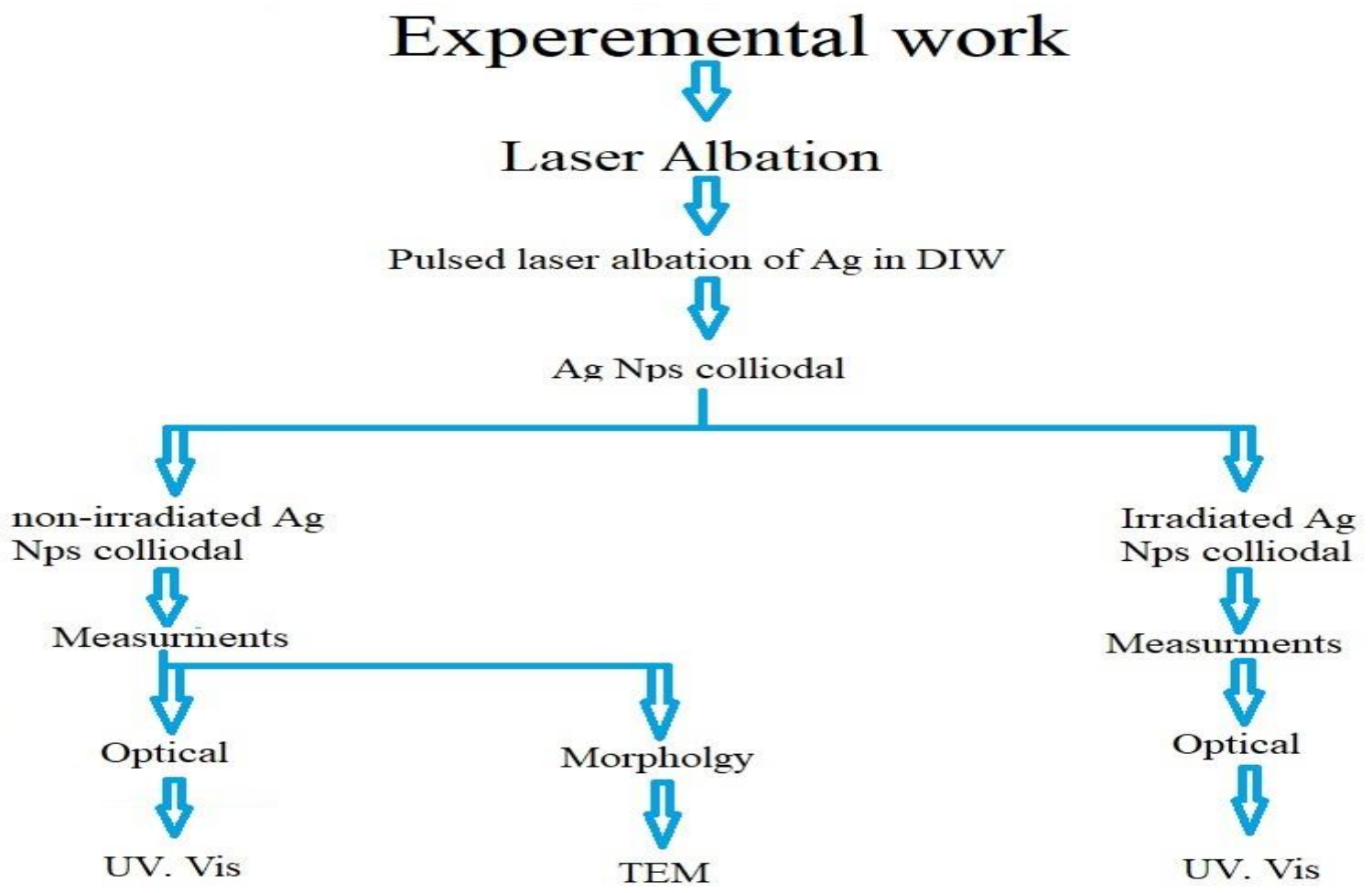

\section{الثكل (2): مخطط توضيحي لعملية الحصول على الجسيمات النانوية.}

يوضح الثكل (3) صورة فونو غر افية لمنظومة الاستئصال بالليزر النبضي للهدف المعدني عالي النقاوة 99.9\% (الفضة) المغمور

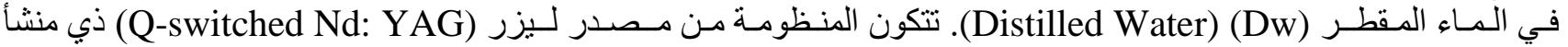

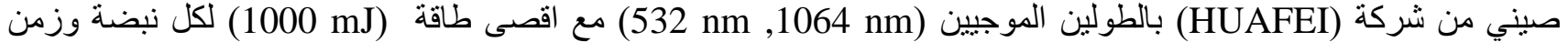

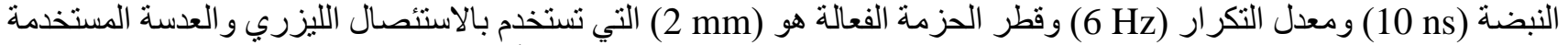

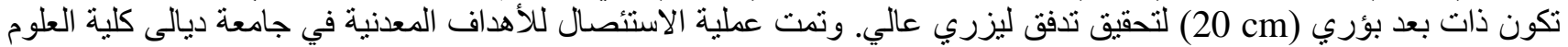

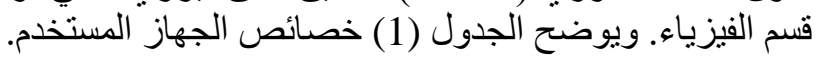

\begin{tabular}{|c|c|}
\hline القيمة & الخاصية \\
\hline $10 \mathrm{~ns}$ & مدة النبض \\
\hline $6 \mathrm{~Hz}$ & معدل التكرار \\
\hline $500 \mathrm{~mJ}-1000 \mathrm{~mJ}$ & طاقة الليزر \\
\hline TEM $_{00}$ & الوضع العرضي \\
\hline Q-switched Nd:YAG & نوع الليزر \\
\hline $532 \mathrm{~nm}-1064 \mathrm{~nm}$ & الطول الموجي \\
\hline
\end{tabular}

تم في هذه الدراسة استخدام هدف معدني من الفضة ذي نقاوة عالية (\% 99.9) وقبل البدء بعملية الاستئصال تصقل سطوح الاهداف

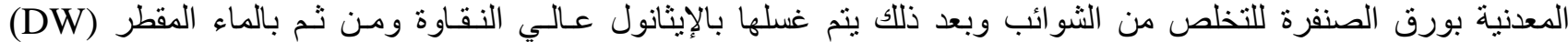

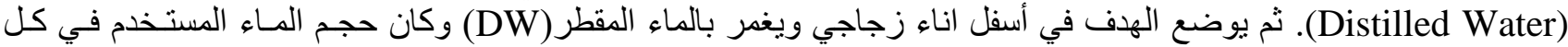

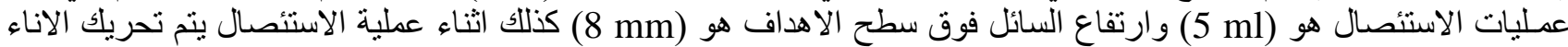

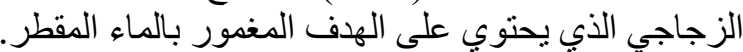

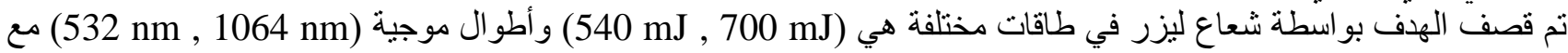
عدد نبضات (100 و 200 و 300 و 400) نبضة. إذ تغير لون الماء بعد عملية الاستئصال و هذا دلالة على تكون الجسيمات 


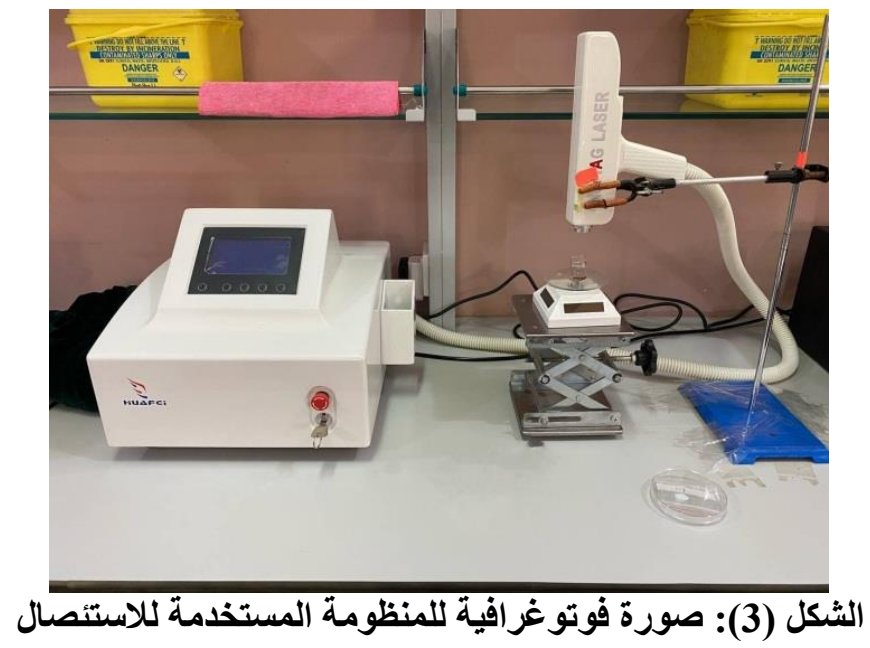

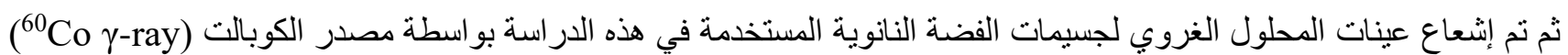

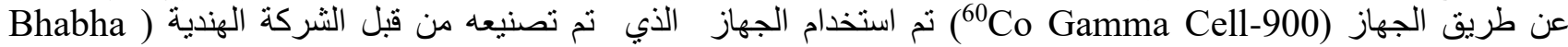
هو (Atomic Research Center / Trombay / Bombay / India

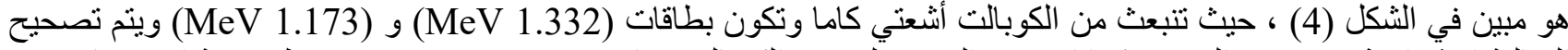

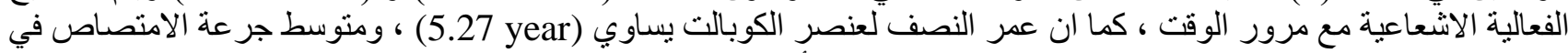

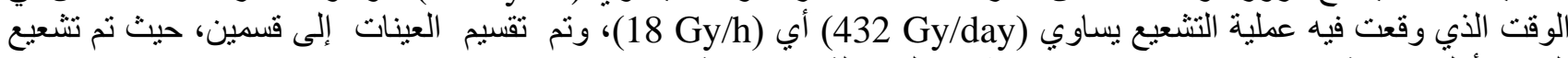

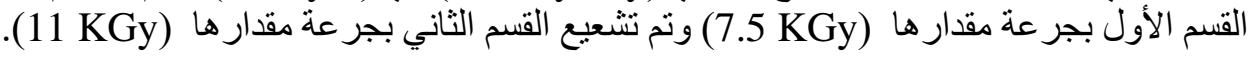

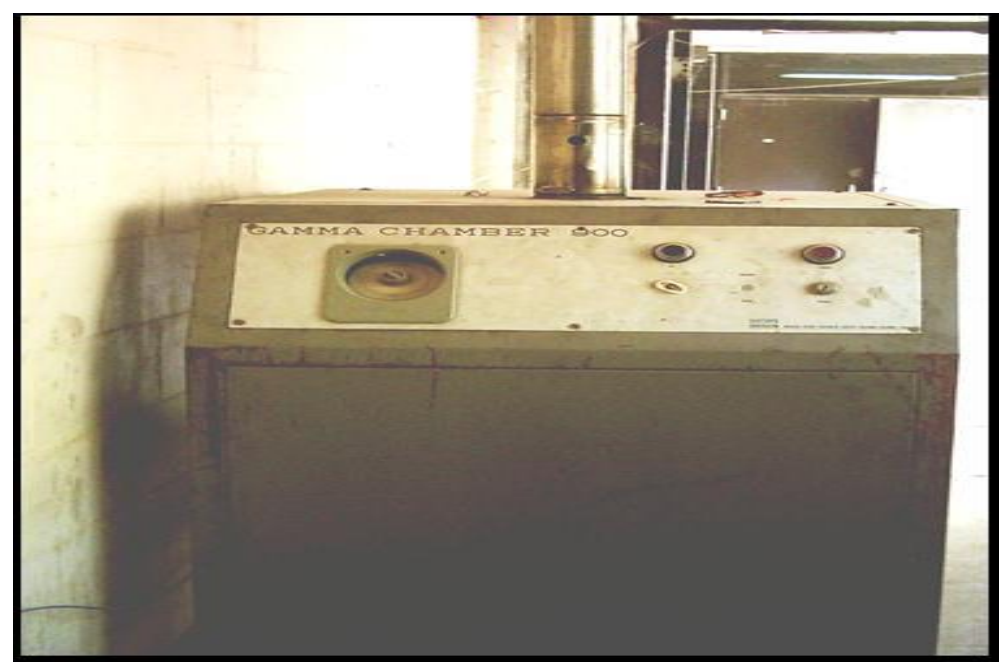

الثكل (4) : جهاز التشعيع Co Gamma Cell-900

وبالنسبة للخصائص البصرية و التشكيلية سيتم در استها من خلال تحقيق بعض القياسات التي تلخص على النحو التالي:-

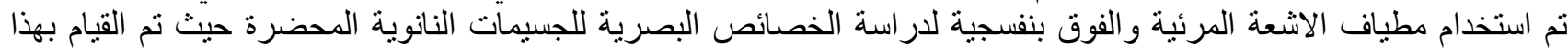

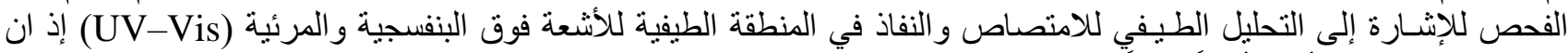

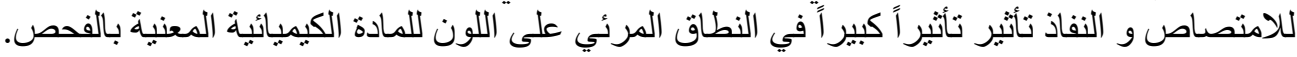

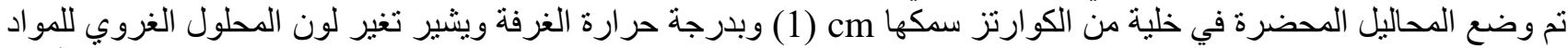

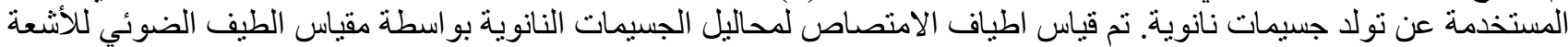

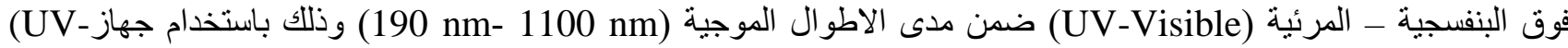
جisible 1800 double beam spectrophotometer) جامعة ديالى/ كلية العلوم/ قسم الفيزياء. و الثنكل(5) يوضح صورة فوتوغر افية للجهاز المستخدم بالفحص لئل 


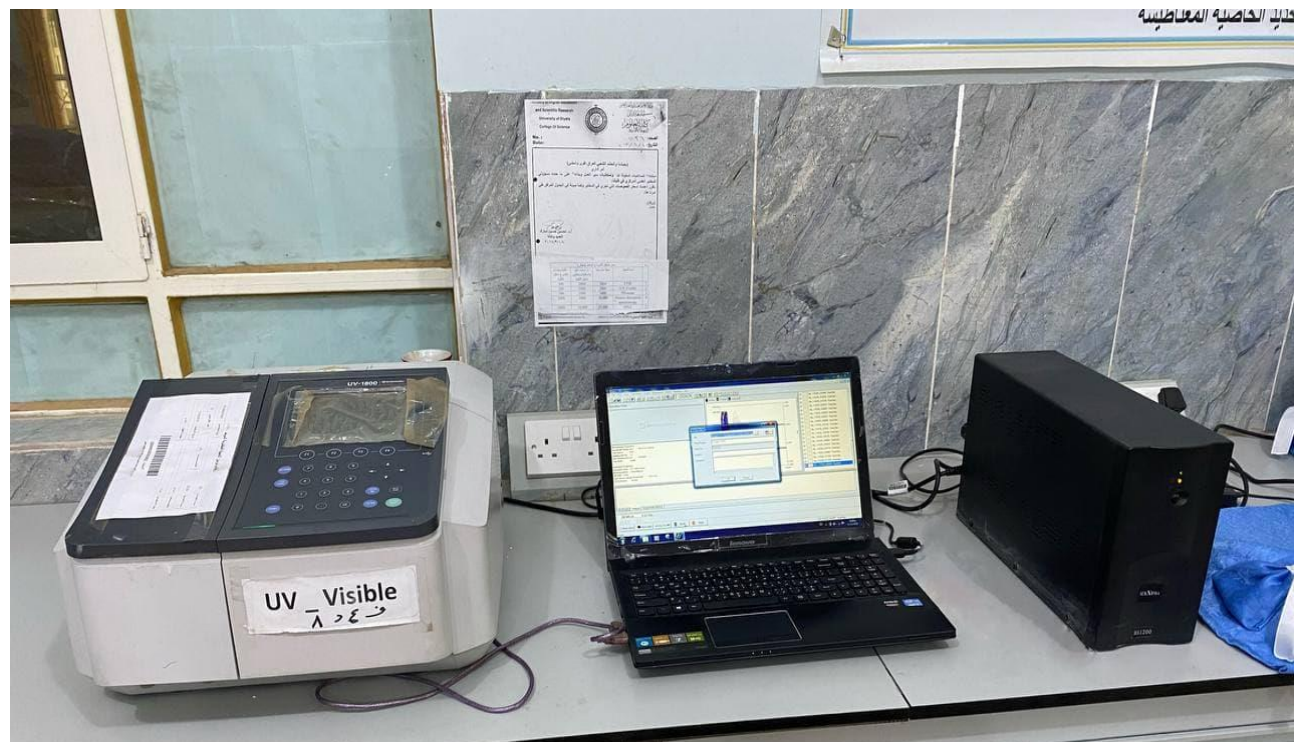

\section{الشكل (5): مطياف الأشعة فوق البنفسجية والمرئية}

ولاجل در اسة الخصائص الموروفولوجية للسطح تم استخدام المجهر الإلكتروني النافذ (TEM) حيث جهاز مهم جدا للبحث في بنية

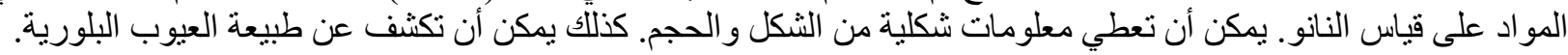

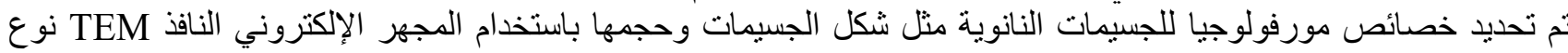
الجئ Carl Zeiss-EM10C-100Kv-Germany الجسيمات النانوية باستخدام برنامج يسمى ImageJ

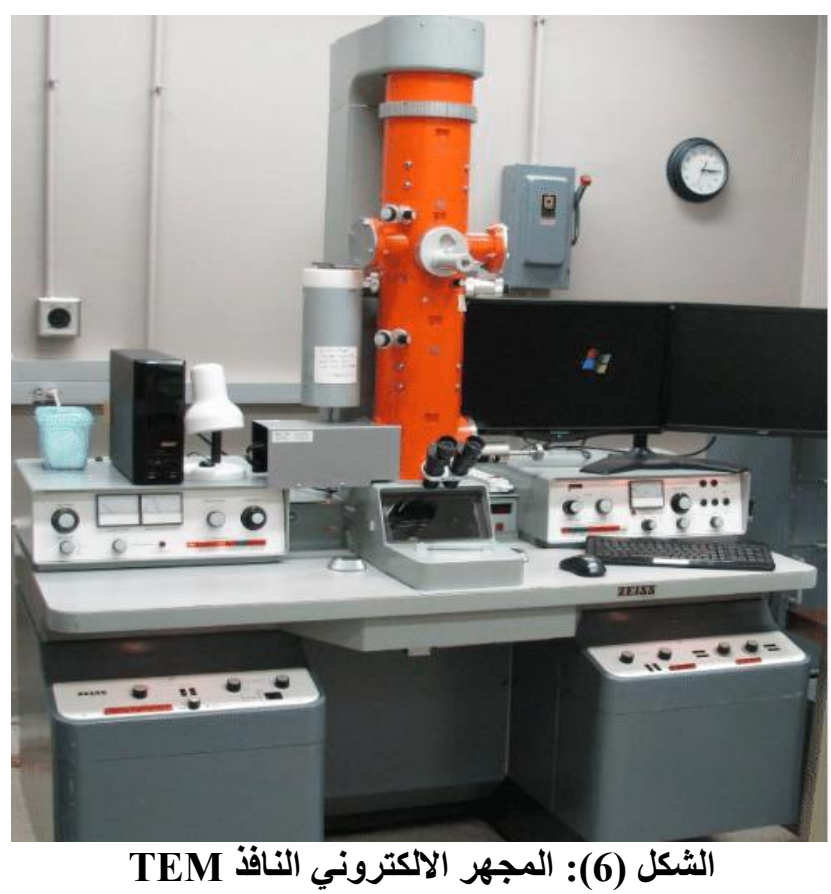

النتائج والمناقشتة

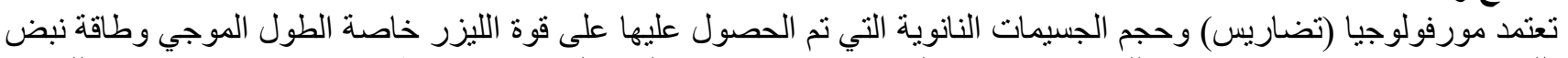
الليزر ـ وتم إجر اء قياسات TEM للتحقيق في مورفولوجيات

الثكل (7) يظهر صورة TEM لجسيمات الفضة النانوية التي يتم تصنيعها بو اسطة الاستئصال بالليزر النبضي في السائل المقطر

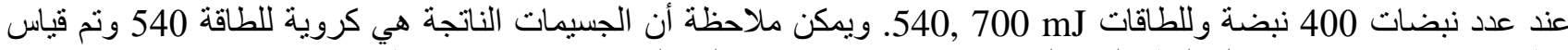

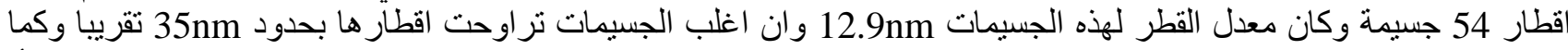
موضح في الثكل (84) التوزيع الاحصائي لهذه الجسيمات. ولكن للطاقة 700 هنالك جسيمات كروية وجسيمات طولية الثكل بهيأة

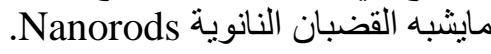




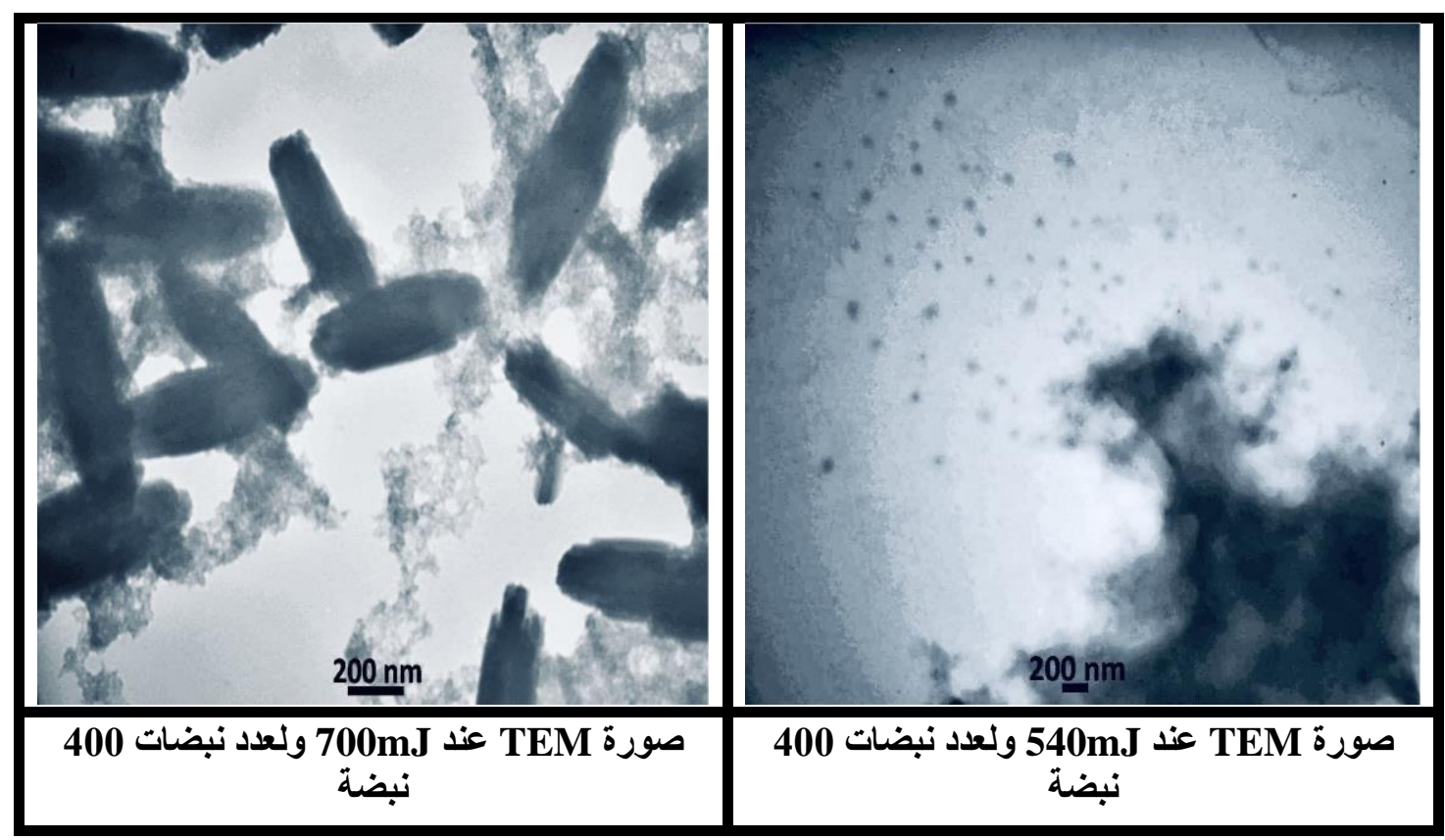

الشكل (7): صورة TEM لجسيمات الفضة النانوية

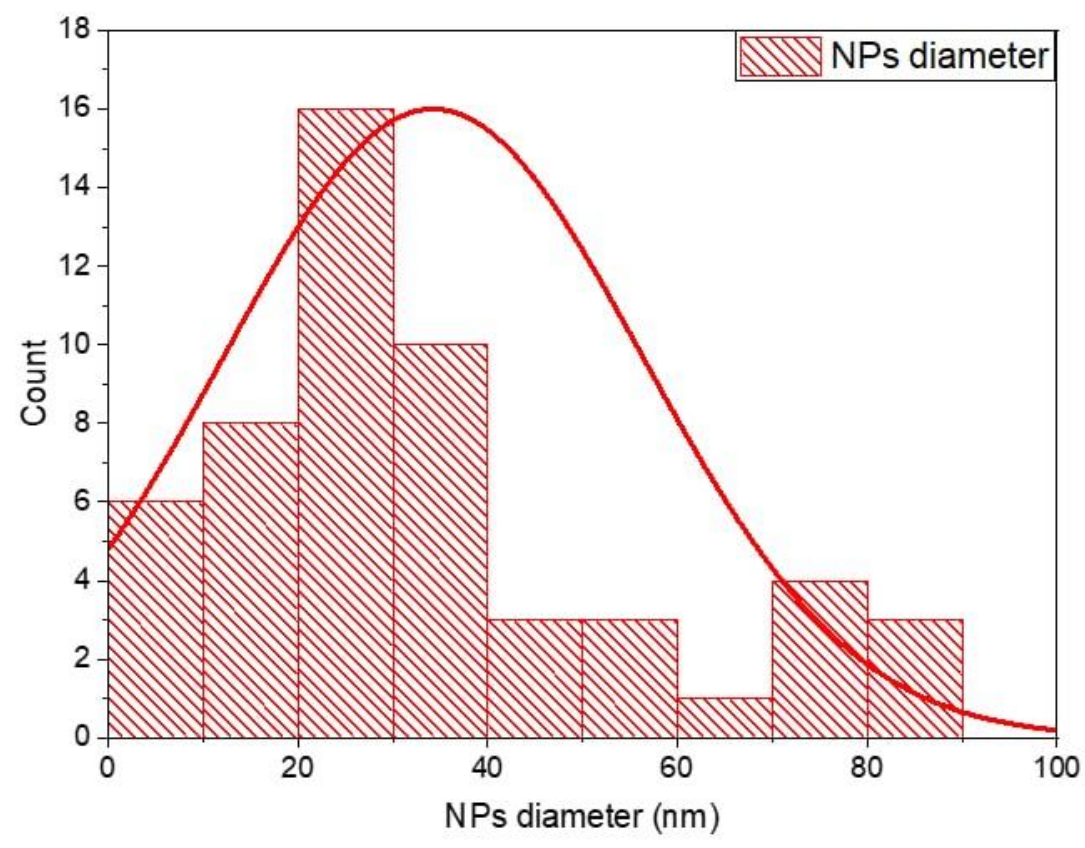

540mJ الثكل (8): التوزيع الاحصائي لاقطار الجسيمات النانوية التي تم الحصول عليها عند طاقة الليزر

أن الخصائص البصرية لها اهمية كبيرة في التحفيز الضوئي فهذه التقنية استخدمت للكثف عن آليات عمل العمليات الضوئية التئية

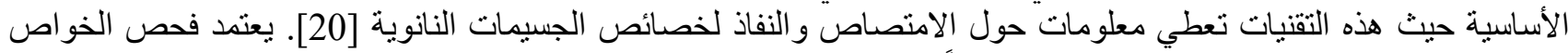

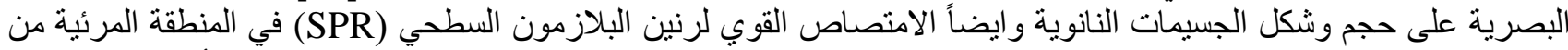

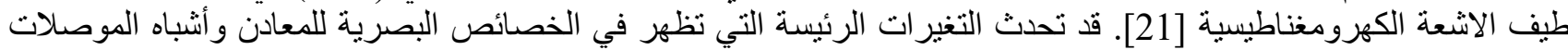
مثل اللون كدالة لحجم الجسيمات حيث يحتوي الهحلول الغروي على جسيمات نانوية فضية لها لون الون أصفر شاحب وتصبح صفر اء

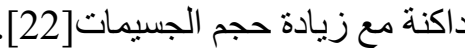

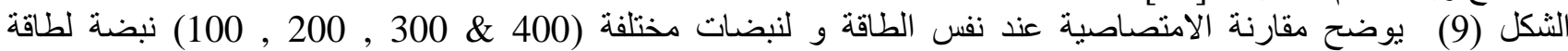
الليزر(5 mJ \& 700 mJ ). وان قمم الامتصاص في المنطقة المرئية هي دلالة المعادن المميزة في تكوين جسيمات نانوية 


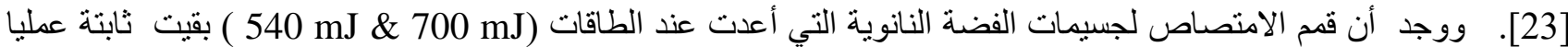

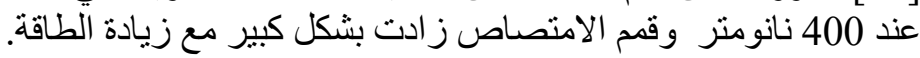

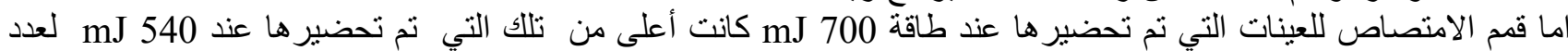

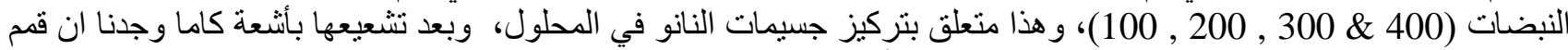

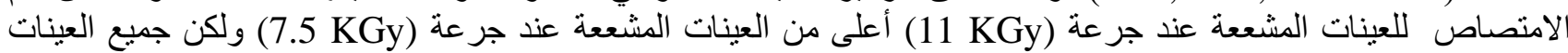
المشععة هي أقل قيمة من العينات غير المشععة في قمم الامتصاص.

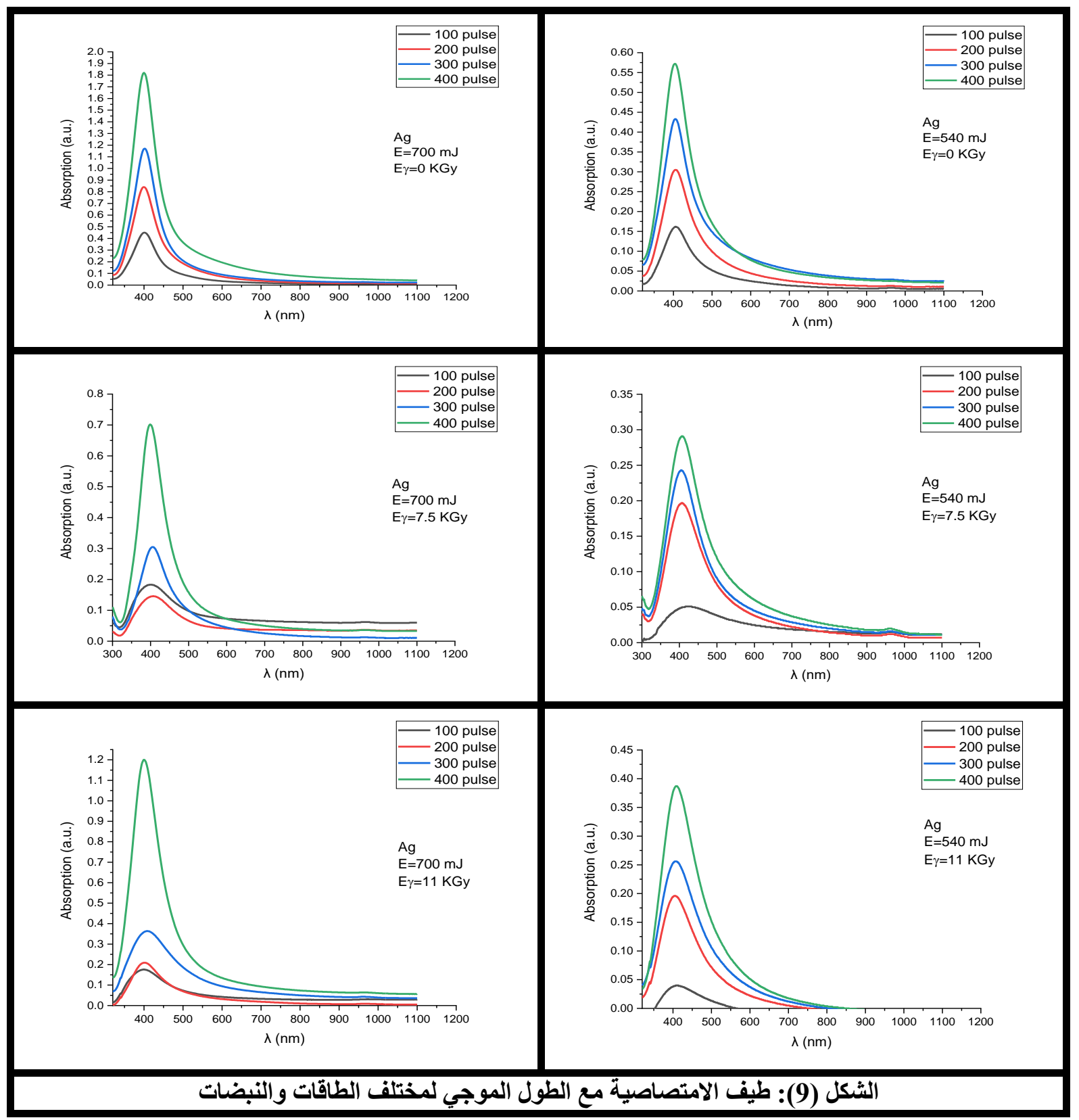

الاسنتناج

تم في هذه الدراسة الحصول على اهم الاستنتاجات التالية:

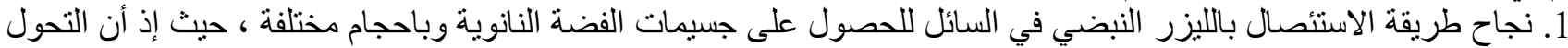

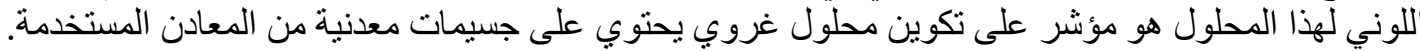
2. يمكن التحكم في حجم الجسيمات وحجم توزيعها بولئ اسطة معلمات الليزر (عدد النبضات والطاقة) ومن خلال الوسط السائل المحيط الذي ينت فيه إجر اء الاستنئصال. 3. أظهر نتائج فحص TEM امكانية الحصول على الألى جسيمات كروية تقريبًا من الفضة. 4. بينت الدر اسة انه كلما ارتفع تركيز المحلول الغرول الغروي ، كلما كان امتصاصده أكبر. 


\section{5. للعينات المشععة امتصاص أعلى بالنسبة لكمية أعلى من الإشعاع في الدراسة لكنها أقل من العينات غير مشععة.}

Acknowledgements

We thank University of Mosul, College of Education for Pure Science, Department of Physics and University of Diyala, College of Science, Department of Physics for supporting this work.

[1] William H. Benson. and Charles M. Auer., Environmental production, United State 100B-07001 (2007).

[2] Rathjen D, L. Read, P. Binks, R.Clark, E. Harvey, B. Innes, C Jagadish, M. Lu, T. Turney, Annual Report, Australia's Research Council. (2009).

[3] Logunov, S.L.; Ahmadi, T.S.; El-Sayed, M.A.; Khoury, J.T.; Whetten, R.L. J. Phys. Chem. B. 101, 19, 3713-3719 (1997).

[4] Burda, C.; Chen, X.; Narayanan, R.; El-Sayed, M.A. Chem. Rev. 105, 4, 1025-1102, (2005)

[5] Bratescu, M.A.; Cho, S.-P.; Takai, O.; Saito, N. J. Phys. Chem. C, 115, 24569-24576, (2011).

[6] Wang, F.; Tang, R.; Yu, H.; Gibbons, P.C.; Buhro, W.E. Chem. Mater. 20, 3656-3662,(2008).

[7] A. Majdalawieh, M. C. Kanan, O. El-Kadri, and S. M. Kanan, Journal of Nanoscience and Nanotechnology, vol. 14, no. 7, pp. 4757-4780, (2014) .

[8] Liz-Marzán, Luis M., Kamat, Prashant V. "Nanoscale Materials" 1st Ed. Kluwer Academic. (2003).

[9] Bohren, C. F. and Huffman D. R. "Absorption and Scattering of Light by Small Particles" 1st Ed. Wiley Company. New York (1983).

[10] Klabunde, K. J., a book published by Wiley-Interscience: New York, 2001.

[11] Sang Hun Lee and Bong-Hyun Jun , International Journal o f Molecular Sciences , 20, 865, (2019).

[12] Navarro. E, Flavio Piccapietra, Bettina Wagner, Fabio Marconi, Ralf Kaegi, Niksa Odzak, Laura Sigg, and Renata Behra. Environ. Sci. Technol., Vol. 42: 8959-8964. (2008).

[13] Laura Carlini, Claudia Fasolato, Paolo Postorino, Ilaria Fratoddi , Iole Venditti, GiovanTesta, Chiara Battocchio, 532, 5, Pages 183-188 , (2017).

[14] Yan Zhao, a Shuanghao Li, Yong Zeng, and Yijian Jiang, AIP Publishing, APL Materials 3, 086103, (2015).

[15] Abdulrahman K. A. Ph.D. Thesis. College of Science, University of Technology, Iraq. (2010).

[16] Wang J B, Zhang C Y, Zhong X L, Yang G W, Chem. Phys. Lett., 361, 86, (2002).

[17] Vincenzo Amendola and Moreno Meneghetti , Phys. Chem. Chem. Phys., 15, 3027, (2013).

[18] Al- Arif, M. Safa, "Biophysics Radiation", 1st Ed. Usama for publishing. Jordan. (1999).

[19] Knoll G. F. "Radiation Detection and measurement", 1st Ed. John Wiley and Sons. New York. (1979).

[20] Khan I , Saeed K., and Khan I, Arabian Journal of Chemistry. 908-931 (2017).

[21] Eustis S. Ph.D. dissertation. College of Science, Georgia Institute of technology, Georgia. (2006).

[22] Thomas P.J., Ph.D. Thesis. Jawaharlal Nehru Centre for Advanced Scientific Research, Bengaluru (2003).

[23] Zheng X, W. Xu, Corredor C., Xu S., An J., J. Phys. Chem. C, 111, 41, 14962-14967 (2007). 